\title{
The effect of chemo- and chemoimmunotherapy on the presence of circulating melanoma cells in peripheral blood. Preliminary results ${ }^{\star \bullet}$
}

\author{
Jolanta Szenajch ${ }^{1}$, Agnieszka Kozak ${ }^{1}$, Adam A. Kruszewski ${ }^{1}$, Ewa Babiej ${ }^{1}$, Małgorzata \\ Chomicka $^{2}$, Jerzy Strużyna ${ }^{2}$ and Wiesław Wiktor-Jędrzejczak ${ }^{1}$ \\ ${ }^{1}$ Department of Immunology and ${ }^{2}$ Department of Reconstructive Surgery, Central Clinical \\ Hospital, Military School of Medicine, Warsaw, Poland
}

Key words: malignant melanoma, reverse transcription/polymerase chain reaction, tyrosinase, chemotherapy, chemoimmunotherapy

\begin{abstract}
Reverse transcription and polymerase chain reaction (RT/PCR) with primers specific for tyrosinase allow for a new method of early detection of individual melanoma cells in peripheral blood. Using this test the effect of chemo- and chemoimmunotherapy on the spread of early micrometastatic cancer cells has been evaluated. No significant correlations have been found between RT/PCR results on the one hand and stage of disease, a kind of the therapy protocol used and usage of the therapy as an adjuvant or palliative on the other hand. Thus, although the RT/PCR test for detection of circulating individual melanoma cells might help in identification of minimal residual disease in some patients, it has no application for routine staging of more advanced disease and in monitoring the response to therapy.
\end{abstract}

In 1994, 1283 new cases of malignant melanoma were registered and 707 persons died from this disease in Poland. Melanoma is placed 20th on the list of newly registered cases of neoplastic diseases in Poland (Zatonski \& Tyczyński, 1997). In 1983-1993, 50\% in- crease in the mortality rate from this disease was observed (J. Didkowska, personal information).

The main problem of melanoma therapy are metastases. They may appear even several years after surgical excision of primary lesion;

\footnotetext{
${ }^{\star}$ Presented at the $6^{\text {th }}$ International Symposium on Molecular Aspects of Chemotherapy, July, 1997, Gdańsk, Poland.

'Supported by the State Committee for Scientific Research (KBN grant No. 4 P05B 059 09)

To whom correspondence should be addressed: Jolanta Szenajch, Department of Immunology, Central Clinical Hospital, Military School of Medicine, Szaserów 128, 00-909 Warsaw; tel: (48 22) 6816298 , 68 16285; tel/fax: (48 22) 6816081.

Abbreviations: AJCC, American Joint Committee on Cancer; bp, base pairs; DTIC, dacarbazine; GAPDH, glyceraldehyde-3-phosphate dehydrogenase; HTYR, human tyrosinase; IL-2, interleukin-2; IFN$\alpha$, interferon $\alpha$; RT/PCR, reverse transcription/polymerase chain reaction.
} 
often they are unresectable and systemic treatment-resistant. The overall median survival time of patients with widespread disease is about 6 months (Lee et al., 1995).

In systemic treatment a single-drug or combination (2-5 drugs) chemotherapy is used. At present (1997) the most active agent applied in chemotherapy is dacarbazine (DTIC), which gives $20 \%$ of partial response rate and can be used in therapy as a single agent (Cocconi et al., 1992). Combination therapies give higher response rates, e.g. DTIC + tamoxifen $-29 \%$ (Cocconi et al., 1992), DTIC + bleomycin + lomustine + vincristine (DOBC) $-32 \%$ (York \& Foltz, 1988) and DTIC + cisplatin + carmustine (or lomustine) + tamoxifen (BCDT) $-46 \%$ (Del Prete et al., 1984).

The combination of chemotherapy with administration of biological response modifiers, e.g. IL-2 and IFN- $\alpha$, increases the antitumor effectiveness of treatment, e.g. combination of cis-platin, IL-2 and IFN- $\alpha$ gives a partial response rate of $54 \%$ (Khayat et al., 1993).

However, these response rates, although significant, have no distinct effect on patients survival and there is no curative therapy known at the advanced stage of disease, so the use of systemic therapy remains palliative.

Therefore, one of the most important goals in modern oncology is to begin treatment of melanoma at the earliest possible stage. To achieve this goal a highly sensitive method for detection of individual neoplastic cells is needed. Such a method would give a chance to diagnose the systemic disease very early before metastases would became recognizable in standard medical examination. A diagnostic test based on this principle might make possible earlier administration of systemic treatment and, possibly, evaluation of the treatment results in individual patients and detection of minimal residual disease (stage M1(i)) defined by the presence of individual tumor cells in secondary organs (Pantel, 1997).

Smith et al. (1991) have described a method for detection of melanoma cells circulating in the blood, based on detection of the transcript for tyrosinase in peripheral blood using RT/PCR. Tyrosinase - one of the melanin biosynthetic enzymes - is present both in normal melanocytes and melanoma cells. However, melanocytes are never present in blood of healthy persons, thus a positive result of this assay indicates the presence of neoplastic cells. This reaction is the most sensitive among all known assays: it can detect a single melanoma cell in $5 \mathrm{ml}$ of blood.

The aim of our study was to evaluate the usefulness of this method in monitoring of chemo- and chemoimmunotherapy.

\section{MATERIALS AND METHODS}

Melanoma cell line. Mew 151, a human melanoma-derived cell line, was established by Dr. Maria Rochowska at The Maria Skłodowska-Curie Memorial Cancer Center in Warsaw (Poland). Cells were cultured in Dulbecco's Modified Eagle Medium (DMEM) with $10 \%$ fetal calf serum (FCS). Cells in the logarithmic growth phase adherent to culture bottle's walls were trypsinized and total RNA was isolated by Chomczynski and Sacchi method (1987).

Patients. Ten healthy blood donors; 5 patients with other neoplasia: chronic lymphatic leukemia (CLL), lymphoma, synovioma malignum, glioblastoma multiforme and breast cancer; 19 patients ( 9 women and 10 men, mean age 55 years, range 24-72) with histologically confirmed malignant melanotic melanoma were examined between January 1995 and May 1997. Seventeen patients had cutaneous melanoma and 2 persons - ocular melanoma as the primary disease. In the period of study the patients were at stage II-IV of disease as evaluated according to AJCC guidelines (1992) (Yeung, 1994): 3 persons at stage II (after excision of primary tumor, $1.6-4 \mathrm{~mm}$ thick, no metastases in regional lymph nodes and no distant metastases), 11 persons - at stage III (after excision of primary tumor, thick $>4 \mathrm{~mm}$, and possible metastases in re- 
gional lymph nodes) and 5 - at stage IV (metastases in regional lymph nodes and distant metastases). Metastases in lymph nodes in patients at stage III were surgically excised in most cases. Patients at stage IV had metastases in the liver ( 1 person), in the brain ( 1 person) and in the lungs ( 3 persons). None of these metastases was resected.

Treatment regimens. Chemo- and chemoimmunotherapy was used: (1) as an adjuvant therapy after surgical excision of primary lesions or solitary metastaises, (2) as a palliative therapy in cases with unresectable metastases.

As a rule, a combination chemotherapy was used. The basic drug in all protocols was DTIC in doses of $250 \mathrm{mg} / \mathrm{m}^{2}$ of total body surface/day during 5 days or $850-1200 \mathrm{mg} / \mathrm{m}^{2}$ of total body surface during 1 day given intravenously. In different protocols DTIC was combined with: 1) tamoxifen (D/T); 2) vincristine and tamoxifen (DVT); 3) vincristine, tamoxifen and hydroxyurea (DVT-HU); 4) vincristine, bleomycine and lomustine (DOBC); 5) vincristine, bleomycine, lomustine and tamoxifen (DOBC-T); 6) vincristine, tamoxifen and lomustine (DOTC); 7) vincristine, bleomycine and tamoxifen (DOBT); 7) lomustine, cis-platin and tamoxifen (BCD-T). Sometimes vincristine was replaced by vinblastine.

One protocol of chemoimmunotherapy was used: $100-200 \mathrm{mg}$ cis-platin was given intravenously during the first day. $18 \times 10^{6} \mathrm{U} /$ day of IL-2 was given subcutaneously from day-2 to day-5, then $9 \times 10^{6} \mathrm{U} /$ day of IFN- $\alpha$ was given from day- 6 to day- 9 . Then the cycle was repeated: IL-2 for six consecutive days and then IFN- $\alpha$ for four consecutive days.

Staging of patients was performed prior to and after therapy according to standard procedures, including physical examination, evaluation of all measurable disease with $\mathrm{X}$ ray, USG and computed tomography.
Blood samples for RT/PCR evaluation were obtained prior to and after therapy not earlier than one week before treatment and not later than one week after treatment and during control visits.

RNA extraction from peripheral blood. For single test, $5 \mathrm{ml}$ of peripheral blood was obtained from each patient, using $125 \mu \mathrm{l}$ of 0.5 M EDTA, pH 8.0, as an anticoagulant. Blood was centrifuged at $400 \times g$ for $6.5 \mathrm{~min}$ and the supernatant was decanted. Total RNA was extracted from cell fraction by Chomczynski and Sacchi method (1987).

RT/nested PCR method. Reactions were performed as described by Brossart et al. (1994), using the reagents and thermo-cycler obtained from Perkin Elmer, and the primers, purchased from the Institute of Hematology in Warsaw. For amplification of tyrosinase mRNA two pairs of primers were used (outer primers: HTYR1 and HTYR2; nested primers: HTYR3 and HTYR4) according to Smith et al. (1991) and for GAPDH mRNA amplification one pair of primers was used according to Brossart et al. (1994). For a single reaction of reverse transcription $0.5 \mu \mathrm{g}$ of total RNA obtained from $5 \mathrm{ml}$ of blood was used. Random hexamers or oligo(dT) were used as primers for reverse transcription. An 1/10 aliquot of the obtained cDNA, was used for GAPDH gene amplification. GAPDH gene expression is constitutive, so that detection of its transcript confirmed good quality of the RNA preparation and proper cDNA synthesis. When the result of GAPDH gene amplification was positive, the remaining aliquots of the obtained $\mathrm{cDNA}$ were used for nested PCR for tyrosinase mRNA. The PCR products were as follows: 319 bp for GAPDH, 284 bp in the first round of PCR (PCR I) and $207 \mathrm{bp}$ in the second round of PCR (PCR II) for tyrosinase. Controls included negative control employing water instead of RNA and positive control employing RNA from Mew 151 cells. 


\section{RESULTS}

Specificity and sensitivity of the RT/PCR method for detection of tyrosinase transcript

GAPDH and tyrosinase transcripts were absent in the negative control, in which water was used instead of RNA. The GAPDH transcript was present in the RNA of blood of all healthy donors and patients with other neoplasia, but the tyrosinase transcript was absent in all of them. Selected representative results are shown in Fig. 1. These data confirm the specificity of the method.

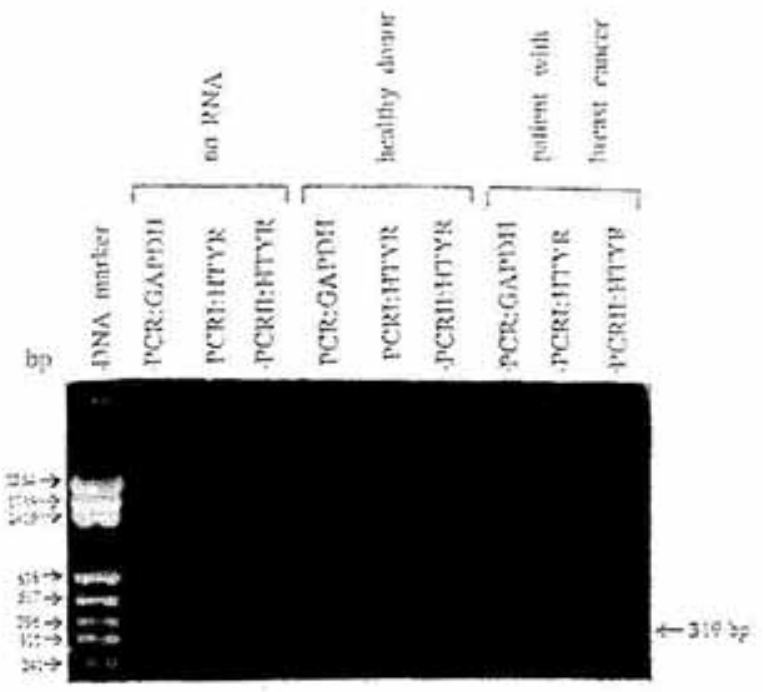

Figure 1. Evaluation of specificity of RT/nested PCR method for detection of tyrosinase transcript in peripheral blood.

PCR products were separated by electrophoresis in $2 \%$ agarose gel.

Total RNA isolated from in vitro cultured Mew 151 cells was found to contain GAPDH and tyrosinase transcripts. In order to determine the sensitivity of the method serial 10 fold dilution experiment was performed. Decreasing numbers of Mew 151 cells $\left(10^{5}-10^{0}\right)$ were mixed with $5 \mathrm{ml}$ of blood from a healthy donor and RNA was extracted. It was found, that in order to obtain a positive band after PCR I, at least $10^{3}$ Mew 151 cells have to be present in the mixture, but only one cell in 5 $\mathrm{ml}$ of blood is sufficient to obtain a positive result after PCR II. Selected representative results are shown in Fig. 2.

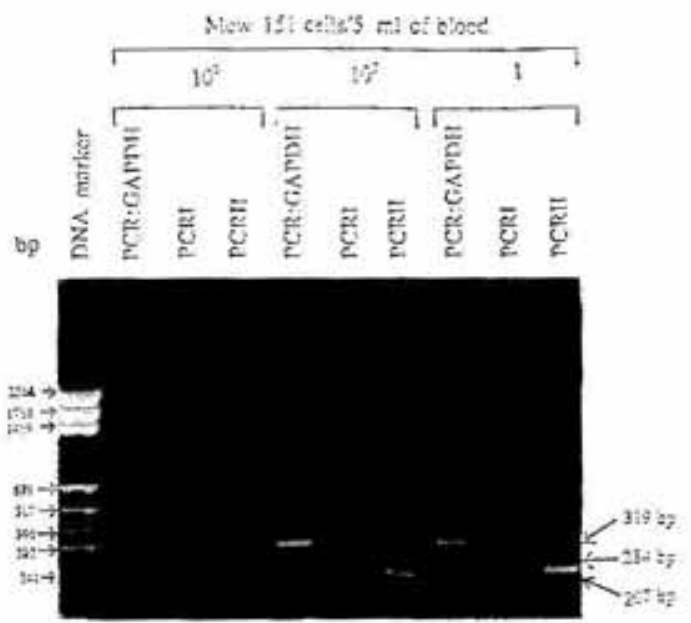

Figure 2. Evaluation of sensitivity of RT/nested PCR method for detection of tyrosinase transcript in peripheral blood.

PCR products were separated by electrophoresis in $2 \%$ agarose gel.

The effect of chemotherapy on the presence of circulating melanoma cells in peripheral blood

Thirty courses of chemotherapy were evaluated. Sixteen patients at the II-IV AJCC stage were included in this study and 12 of them were examined several $(2-5)$ times prior to and after consecutive courses of treatment.

All four possible situations were observed during the examinations:

1) tyrosinase transcript was absent in the blood prior to as well as after the course of therapy $(-/-)$,

-2) tyrosinase transcript was present in the blood prior to as well as after the course of therapy $(+/+)$,

3) tyrosinase transcript was absent in the blood prior to the course of therapy and present afterwards $(-/+)$,

4) tyrosinase transcript was present in the blood prior to the course of therapy and absent afterwards $(+/-)$, 
Four selected cases, which illustrate these four situations, are shown in Fig. 3.

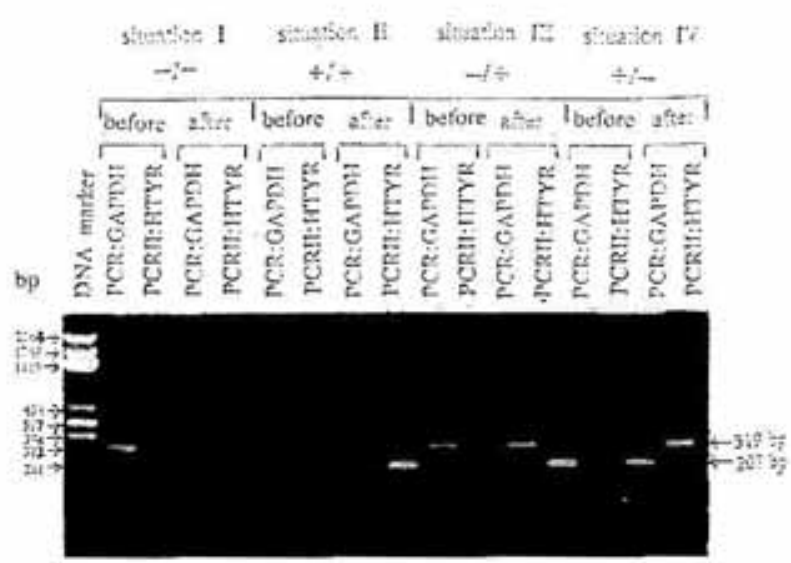

Figure 3. Different effects of therapy indicated by the presence of tyrosinase transcript in peripheral blood - four observed situations.

PCR products were separated by electrophoresis in $2 \%$ agarose gel. Signs + and - denote the presence or absence, respectively, the tyrosinase transcript in the blood of patients, e.g. $+/$ - denotes the presence of tyrosinase transcript in the blood of patient before the therapy and absence of it after the course.

Summary of the results is presented in Table 1. $\mathrm{RT} / \mathrm{PCR}$ results in relation to AJCC stage of patients are shown in Table 2.

The RT/PCR results in relation to protocols of therapy used in the study are shown in Table 3. Only a few courses of each protocol were evaluated, therefore the data are insufficient to permit any generalization. It can only be noted that the effect of the same protocol very

Table 1. Summary of RT/PCR results prior to and after chemotherapy in patients with melanoma.

For details see text.

\begin{tabular}{lllll}
\hline Number of evaluated courses & RT/PCR results \\
\hline & $-/ /-+/+$ & $-/+$ & $+/-$ \\
30 & 9 & 9 & 5 & 7 \\
\hline
\end{tabular}

Signs + and - denote the presence or absence, respectively, the tyrosinase transcript in the blood of patients, e.g. + / denotes the presence of tyrosinase transcript in the blood of patient before the course of therapy and absence of it after the course. often differed from one particular courses to another.

Table 2. RT/PCR results prior to and after chemotherapy courses in relation to AJCC stage of patients with melanoma.

For details see text.

\begin{tabular}{|c|c|c|c|c|c|}
\hline \multirow[t]{2}{*}{ AJCC stage } & \multicolumn{4}{|c|}{$\mathrm{RT} / \mathrm{PCR}$ results } & \multirow{2}{*}{$\begin{array}{l}\text { Total } \\
\text { number } \\
\text { of courses }\end{array}$} \\
\hline & $-1-$ & $+1+$ & $-1+$ & $+/-$ & \\
\hline II & 2 & 0 & 1 & 0 & 3 \\
\hline III & 3 & 6 & 3 & 5 & 17 \\
\hline IV & 4 & 3 & 1 & 2 & 10 \\
\hline $\begin{array}{l}\text { Total number } \\
\text { of courses }\end{array}$ & 9 & 9 & 5 & 7 & 30 \\
\hline
\end{tabular}

Signs + and -, as described in a footnote to Table 1.

$\mathrm{RT} / \mathrm{PCR}$ results prior and after therapy in patients treated with adjuvant and palliative chemotherapy are shown in Table 4.

RT/PCR results prior and after chemotherapy in some selected cases are shown in Table 5. As it is seen, the administration of chemotherapy according to a particular protocol often gave different effects regarding the appearance/disappearance of the tyrosinase transcript in the same patient.

The effect of chemoimmunotherapy on the presence of circulating melanoma cells in peripheral blood

Three courses of chemoimmunotherapy were evaluated.

Patient No. 5 at stage III tyrosinase positive prior to the course of palliative chemoimmunotherapy became negative after treatment.

In two patients (No. 6 at II and No. 7 at stage III), in whom therapy courses were adjuvant, the results were opposite: negative prior to and positive after therapy. The therapy course was interrupted in the patient No. 6 after 5 days because of severe side effects. Patient No. 7 was evaluated three times during the course and each time he was positive. The schedule of treatment and RT/PCR tests in 
Table 3. RT/PCR results prior to and after chemotherapy in relation to the therapy protocol administered to patients with melanoma

\begin{tabular}{lllllc}
\hline \multirow{2}{*}{ Chemotherapy protocol } & \multicolumn{3}{c}{ RT/PCR results } & Total number of \\
\cline { 2 - 6 } & $-/-$ & $+/+$ & $-/+$ & $+/-$ & courses \\
\hline D/T & 0 & 0 & 1 & 2 & 3 \\
DVT & 4 & 2 & 0 & 1 & 7 \\
DVT-HU & 0 & 0 & 1 & 1 & 2 \\
DOBC & 3 & 0 & 0 & 1 & 4 \\
DOBC-T & 1 & 2 & 2 & 2 & 7 \\
DOTC & 1 & 0 & 1 & 0 & 2 \\
DOBT & 0 & 4 & 0 & 0 & 4 \\
BCD-T & 0 & 1 & 0 & 0 & 1 \\
\hline Total number & 9 & 9 & 5 & 7 & 30 \\
of courses & & & &
\end{tabular}

D/T, tamoxifen; DVT, vincristine and tamoxifen; DVT-HU, vincristine, tamoxifen and hydroxyurea; DOBC, vincristine, bleomycine and lomustine; DOBC-T, vincristine, bleomycine, lomustine and tamoxifen; DOTC, vincristine, tamoxifen and lomustine; DOBT, vincristine, bleomycine and tamoxifen; BCD-T, lomustine, cis-platin and tamoxifen.

Signs + and -, as described in a footnote to Table 1.

this patient are presented in Table 6. Three weeks after this chemoimmunotherapy course metastases were found in the patient's lung and liver. Unfortunately, both these cases represented patients in whom the disease progressed during the therapy and none of the patients who responded to therapy was monitored by this method.

Table 4. RT/PCR results prior to and after therapy in patients with melanoma treated with adjuvant or palliative chemotherapy

\begin{tabular}{llllll}
\hline Chemotherapy & \multicolumn{3}{c}{ RT/PCR results } & $\begin{array}{l}\text { Total } \\
\text { number of } \\
\text { courses }\end{array}$ \\
\cline { 2 - 5 } & $-1-$ & $+/+$ & $+/-$ & $+/-$ & \\
\hline Adjuvant & 3 & 6 & 3 & 5 & 17 \\
$\begin{array}{l}\text { Palliative } \\
\text { Total number } \\
\text { of courses }\end{array}$ & 6 & 3 & 2 & 2 & 13 \\
\hline
\end{tabular}

Signs + and - , as described in a footnote to Table 1.

\section{DISCUSSION}

In this study we approached the problem of monitoring the response to therapy in melanoma using the RT/PCR method for detection of single circulating neoplastic cells. No significant correlations have been found between the effects of therapy on the level of circulating melanoma cells on the one hand, and the
AJCC stage of disease, a kind of used protocol and usage therapy as an adjuvant or palliative on the other hand. Whether this reflects differences in biology of the disease in individual patients or differences in their response to therapy is unclear.

In our study we have observed all possible situations: melanoma cells disappeared from the blood of some patients after courses of therapy, in other patients they appeared after courses of therapy or sometimes the administered therapy had no influence on the presence of tumor cells. These results are in conflict with the results described by Brossart et al. (1994): in their work the tyrosinase transcript was detected in blood and bone marrow samples from 28 patients with malignant melanoma at stage III and IV prior to and after immmunotherapy with IL-2 and IFN- $\alpha$. The authors in their subsequent investigations found that many patients were negative after chemotherapy (U. Keilholz, personal information). It is difficult to explain these differences and more extended evaluation of melanoma patients is necessary to find out the reason.

On the one hand, the observed situations of appearance or persistence of the tyrosinase transcript after treatment could represent a further confirmation of poor efficacy of sys- 
Table 5. RT/PCR results prior to and after chemotherapy in some selected cases of melanoma.

For the explanation of abbreviations see a legend to Fig. 3.

\begin{tabular}{llll}
\hline Patient No. & AJCC stage & $\begin{array}{l}\text { Number of courses } \\
\text { chemotherapy protocol }\end{array}$ & $\begin{array}{l}\text { RT/PCR results prior to and } \\
\text { after consecutive courses of } \\
\text { therapy }\end{array}$ \\
\hline 1 & III & $2 \times$ DOBT & $+/+;+/+$ \\
2 & III & $2 \times$ DOBC-T & $+/-;-/+$ \\
3 & IV & $2 \times$ DVT-HU & $+/-;-/+$ \\
4 & III & $3 \times$ DVT & $+/-;-/-;-1-$ \\
\hline
\end{tabular}

Signs + and - , as described in a footnote to Table 1.

temic therapy in advanced melanoma. On the other hand, the difficulties in interpretation of results could be caused by the fact that examination by employing the RT/PCR method does not answer some important questions. First, the assay is only qualitative, so that in some situations the results cannot be interpreted clearly, e.g. in the case of the result $+/+$ it is not known whether the number of melanoma cells increased, decreased or remained the same after treatment. Developing the quantitative PCR might help to resolve this problem.

Table 6. RT/PCR results before, during and after chemoimmunotherapy in patient No. 7

\begin{tabular}{ll}
\hline Schedule of the course & RT/PCR results \\
\hline $\begin{array}{l}\text { day-0 - before therapy } \\
\text { day-1: cis-platin }\end{array}$ & negative \\
$\begin{array}{l}\text { day-2 - day- } 5: \text { IL-2 } \\
\text { day-7, day- } 8 \text { : INF- } \alpha\end{array}$ & \\
day-10: & positive \\
day-11 - day-13: INF- $\alpha$ & \\
day-14: IL-2 & positive \\
$\begin{array}{l}\text { day-15 - day-18: IL-2 } \\
\text { day-19: IL-2 }\end{array}$ & positive \\
$\begin{array}{l}\text { day-20, day-21, day-23, } \\
\text { day-24: INF- } \alpha\end{array}$ & \\
day-30 - after therapy & positive \\
\hline
\end{tabular}

Second, the result of this test does not provide information about the metastatic potential of circulating melanoma cells, because this potential depends on the expression of genes other than tyrosinase (Hoon et al., 1995). It is known that only $0.01 \%$ of circulating cancer cells are capable to establish metastasis (Fidler, 1990). This ability depends on multiple parameters, which include, among others, 1) the tumor's cell capacity for traversing basement membrane, which requires expression of collagenase type IV (Seftor et al., $1990), 2$ ) interaction with host cells - an inverse relationship between patients' survival and serum level of intercellular adhesion molecule (ICAM-1) has been reported in melanoma (Harning et al., 1991), 3) melanoma cell survival during circulation in blood, 4) potential of growth and colonization of distant organs, dependent on expression of numerous growth factors and immunomodulators (e.g. transforming growth factor- $\alpha$ and $\beta$, basic fibroblast growth factor, plateled-derived growth factor, interleukin- $1 \alpha$ and $\beta$, interleukin-6, monocyte chemotactic and activating factor and granulocyte-macrophage colony stimulating factor (Lupetti et al., 1996; Rodeck \& Herlyn, 1991; Singh et al., 1995) and by capacity of tumor cells to induce angiogenesis in the place of colonization (Barnhill et al., 1992). Therefore the presence of circulating melanoma cells, identified by detection of the tyrosinase transcript, indicates only that melanoma cells are capable of entering the circulation from a primary or secondary tumor but gives no information concerning their capacity to leave the circulation and to initiate a metastasis. 


\section{REFERENCES}

Barnhill, R.L., Fandrey, K., Levy, M.A., Mihm, M.C., Jr. \& Hyman, B. (1992) Angiogenesis and tumor progression of melanoma. Quantification of vascularity in melanocytic nevi and cutaneous malignant melanoma. Lab. Invest. 67, 331-337.

Brossart, P., Keilholz, U., Scheibenbogen, C., Möhler, T., Willhauck, M. \& Hunstein, W. (1994) Detection of residual tumor cells in patients with malignant melanoma responding to immunotherapy. J. Immunol. 15, 38-41.

Chomczynski, P. \& Sacchi, N. (1987) Single-step method of RNA isolation by acid guanidinium thiocyanate-phenol-chloroform extraction. Anal. Biochem. 162, 156-159.

Cocconi, G., Bella, M., Calabresi, F., Tonato, M., Canaletti, R., Boni, C., Buzzi, F., Ceci, G., Corgina, E., Costa, P., Lottici, R., Papadia, F., Sofra, M.C. \& Baccchi, M. (1992) Treatment of metastatic malignant melanoma with dacarbazine plus tamoxifen. $N$. Engl. J. Med. 327, 516-523.

Del Prete, S.A., Maurer, L.H., O'Donnel, J., Forcier, R.J. \& Lemarbre, P. (1984) Combination chemotherapy with cisplatin, carmustine, dacarbazine and tamoxifen in malignant melanoma. Cancer Treat. Rep. 68, 1403-1405.

Fidler, I.J. (1990) Critical factors in the biology of human cancer metastasis: Twenty-Eigth G.H.A. Clowes Memorial Award Lecture. Cancer Res. 50, 6130-6138.

Harning, R., Mainolfi, E., Bystryn, J.C., Henn, M., Merluzzi, V.J. \& Rothlein, R. (1991) Serum levels of circulating adhesion molecule 1 in human malignant melanoma. Cancer Res. 51, 5003-5005.

Hoon, D.S.B., Wang, Y., Dale, P.S., Conrad, A.J., Schmid, P., Garrison, D., Kuo, Ch., Fashang, L.J., Nizze, A.J. \& Morton, D.L. (1995) Detection of occult melanoma cells in blood with a multiple-marker polymerase chain reaction assay. J. Clin. Oncol. 13, 2109-2116.
Khayat, D., Borel, C. \& Tourani, J.M., Benhammouda, A., Antoine, E., Rixe, O., Vuillemin, E., Bazex, P.A., Thill, L. \& Franks, R. (1993) Sequential chemoimmunotherapy with cisplatin, interleukin-2 and interferon-alfa-2a for metastatic melanoma. J. Clin. Oncol. 11, 2173-2180.

Lee, S.M., Betticher, D.C. \& Thatcher, N. (1995) Melanoma: chemotherapy. Brit. Med. Bull. 51, 609-630.

Lupetti, R., Mortarini, R., Panceri, P., Sensi, M. \& Anichini, A. (1996) Interaction with fibronectin regulates cytokine gene expression in human melanoma cells. Int. J. Cancer 66, 110-116.

Pantel, K. (1997) Detection of minimal residual disease in patients with epithelial cancer. ABMTR Newslett. 14, 1-5.

Rodeck, U. \& Herlyn, M. (1991) Growth factors in melanoma. Cancer Metastasis Rev. 10, 89-101.

Seftor, E.A., Seftor, R.E.B. \& Hendrix, M.J.C. (1990) Selection of invasive and metastatic subpopulation from a heterogenous human melanoma cell line. BioTechniques 9, 324-331.

Singh, R.K., Gutman, M. \& Radinsky, R. (1995) Heterogenity of cytokine and growth factor gene expression in human melanoma cells with different metastatic potentials. $J$. Int. Cyt. Res. 15, 81-87.

Smith, B., Selby, P., Southgate, J., Pittman, K., Bradley, Ch. \& Blair, G.E. (1991) Detection of melanoma cells in peripheral blood by means of reverse transcriptase and polymerase chain reaction. Lancet 338, 1227-1229.

York, R.M. \& Foltz, A.T. (1988) Bleomycin, vincristine, lomustine and DTIC chemotherapy for metastatic melanoma. Cancer 61, 2183-2186.

Zatoński, W. \& Tyczyński, J. (eds.) (1997) Cancer in Poland in 1994, The M. Sklodowska-Curie Memorial Cancer Center, National Cancer Registry, Warszawa. 